\title{
Late complications following permanent pacemaker implantation or elective unit replacement
}

\author{
A A Harcombe, S A Newell, P F Ludman, T E Wistow, L D Sharples, P M Schofield, \\ D L Stone, L M Shapiro, T Cole, M C Petch
}

Regional Cardiac Unit, Papworth Hospital NHS Trust, Cambridge CB3 8RE, UK

A A Harcombe

$S$ A Newell

P F Ludman

T E Wistow

P M Schofield

D L Stone

L M Shapiro

T Cole

M C Petch

MRC Biostatistics Unit, Research and Development Unit, Papworth Hospital NHS Trust

L D Sharples

Correspondence to: Dr Petch.

Accepted for publication 6 April 1998

\begin{abstract}
Objective-To determine the rate of late complications following first implantation or elective unit replacement of a permanent pacemaker system.

Design-Analysis of pacemaker data and complications prospectively acquired on a computerised database. Complications were studied over an 11 year period from January 1984 to December 1994.

Setting-Tertiary referral cardiothoracic centre.

Patients-Records of 2621 patients were analysed retrospectively.

Main outcome measures-Complications requiring repeat procedures occurring more than six weeks after pacemaker implantation or elective unit replacement. Results-The overall rate of late complications was significantly lower after first implantation of a permanent pacemaker (34 cases, complication rate $1.4 \%, 95 \%$ confidence interval $0.9 \%$ to $1.9 \%$ ) than after elective unit replacement (16 cases, complication rate $6.5 \%(3.3 \%$ to $9.7 \%)$. There were 20 cases of erosion, 18 infections, five electrode problems, and seven miscellaneous problems. Complications were more common with inexperienced operators $(18.9 \%(6.0 \%$ to $31.8 \%))$ than with experienced operators $(0.9 \%(0.3 \%$ to $1.5 \%)$ ).

Conclusions-The incidence of late complications following pacemaker implantation is low and compares favourably with early complication rates. The majority are caused by erosion and infection. Patients who have undergone elective unit replacement are at particular risk.

(Heart 1998;80:240-244)
\end{abstract}

Keywords: permanent pacemaker; elective unit replacement; late complications; arrhythmias

We have previously reported a prospective study of early complications following single chamber permanent pacemaker implantation. ${ }^{1}$ Mugica et al reported a similar study. ${ }^{2}$ Further studies from our unit and others examined early complication rates for single versus dual chamber systems. ${ }^{3}$ The results of these studies suggested a revision of the level at which early complication rates were deemed acceptable, ${ }^{5}$ from less than $5 \%$ down to less than $3 \%$. There are, however, no data specifically addressing late complications after permanent pacemaker implantation.
We therefore conducted a further study to determine the incidence and nature of late complications following permanent pacemaker implantation. We assessed both dual and single chamber pacemakers and also studied complications following elective unit replacement.

\section{Methods}

PATIENTS

During the period from 1 January 1984 to 31 December 1994, 2621 patients had pacemaker implantation or elective unit replacement (EUR) for end of battery life; 1538 (59\%) were male (mean age 73 years) and 1083 (41\%) were female (mean age 76 years). Of the systems implanted, 2220 were single chamber (including 235 EUR) and 401 dual chamber (10 EUR).

\section{PROCEDURES}

All pacemaker implantation data are prospectively entered on to a custom designed computer database. The following information is

Table 1 Operator experience and number of complications

\begin{tabular}{llcl}
\hline Operator & Experience & Procedures & Complications \\
\hline 1 & EI & 3 & 1 \\
2 & EI & 4 & 1 \\
3 & $\mathrm{I}$ & 5 & 1 \\
4 & $\mathrm{I}$ & 6 & 1 \\
5 & $\mathrm{I}$ & 8 & 1 \\
6 & $\mathrm{I}$ & 8 & 2 \\
7 & $\mathrm{I}$ & 10 & 2 \\
8 & $\mathrm{EI}$ & 17 & 0 \\
9 & $\mathrm{E}$ & 25 & 0 \\
10 & $\mathrm{EI}$ & 31 & 2 \\
11 & $\mathrm{E}$ & 31 & 0 \\
12 & $\mathrm{~T}$ & 35 & 0 \\
13 & $\mathrm{~T}$ & 72 & 2 \\
14 & $\mathrm{~T}$ & 81 & 1 \\
15 & $\mathrm{EI}$ & 82 & 0 \\
16 & $\mathrm{~T}$ & 82 & 1 \\
17 & $\mathrm{~T}$ & 83 & 4 \\
18 & $\mathrm{~T}$ & 90 & 0 \\
19 & $\mathrm{~T}$ & 93 & 0 \\
20 & $\mathrm{E}$ & 96 & 1 \\
21 & $\mathrm{~T}$ & 101 & 2 \\
22 & $\mathrm{~T}$ & 114 & 3 \\
23 & $\mathrm{~T}$ & 118 & 3 \\
24 & $\mathrm{EI}$ & 122 & 1 \\
25 & $\mathrm{~T}$ & 133 & 2 \\
26 & $\mathrm{~T}$ & 139 & 5 \\
27 & $\mathrm{~T}$ & 139 & 2 \\
28 & $\mathrm{~T}$ & 142 & 1 \\
29 & $\mathrm{E}$ & 147 & 2 \\
30 & $\mathrm{E}$ & 174 & 3 \\
31 & $\mathrm{E}$ & 179 & 0 \\
32 & $\mathrm{E}$ & 219 & 2 \\
$?$ & $?$ & 32 & 4 \\
Total & & 2621 & 50 \\
& & &
\end{tabular}

The number of procedures performed during the study are listed in ascending order alongside the number of complications that occurred.

E, experienced; EI, experienced infrequent operator; T, undergoing training; I, inexperienced and performed $<12$ procedures during the study. 
Table 2 Number of complications in the late period following pacemaker implantation or elective unit replacement

\begin{tabular}{|c|c|c|c|c|c|}
\hline & Infection & Erosion & $\begin{array}{l}\text { Electrode } \\
\text { problems }\end{array}$ & Miscellaneous & Totals \\
\hline \multicolumn{6}{|l|}{ First implants } \\
\hline $\begin{array}{c}\text { Single chamber } \\
(\mathrm{n}=1985)\end{array}$ & $9(0.4 \%)$ & $8(0.4 \%)$ & $3(0.2 \%)$ & $5(0.2 \%)$ & $25(1.3 \%)$ \\
\hline $\begin{array}{l}\text { Dual chamber } \\
(\mathrm{n}=391)\end{array}$ & $4(1 \%)$ & $2(0.5 \%)$ & $1(0.3 \%)$ & $2(0.5 \%)$ & $9(2 \%)$ \\
\hline $\begin{array}{l}\text { EUR }(\mathrm{n}=245 ; 10 \text { dual } \\
\text { chamber) }\end{array}$ & $5(1.6 \%)$ & $10(4.1 \%)$ & $1(0.4 \%)$ & 0 & $16(6.5 \%)$ \\
\hline Total $(n=2621)$ & $18(0.7 \%)$ & $20(0.8 \%)$ & $5(0.2 \%)$ & $7(0.3 \%)$ & $50(1.9 \%)$ \\
\hline
\end{tabular}

EUR, elective unit replacement.

stored: patient details, indications for pacing, operator, venous access route, pacemaker generator, electrode type, and sensing and pacing thresholds. Subsequent complications are recorded on the same database. All single and dual chamber pacemaker implantations and EUR procedures during the study period (11 years) were analysed for complications occurring up to one year after the last implantation (that is, up to 31 December 1995). Late complications were defined as those occurrences requiring operative intervention later than six weeks after the procedure. Complications which could be dealt with by reprogramming were not included. In studies of early complications the first six weeks postimplantation have been used to define the early period, except in that of Aggarwal et al, where the first two months were studied. ${ }^{4}$ Unit replacements required as a result of complications (early or late) were excluded. The definitions used in this study have been described previously. ${ }^{1}$

Implantation was performed in the radiology department under local anaesthetic after premedication with an oral benzodiazepine. From January 1984 to February 1990, no antibiotics were given. From March 1990, patients were treated with oral antibiotics (flucloxacillin, or erythromycin in case of allergy) for 48 hours, the first dose given immediately before the

Table 3 Positive microbiological findings

\begin{tabular}{|c|c|c|c|}
\hline Complication & Case & Organism & Source \\
\hline \multirow[t]{11}{*}{ Infection } & 1 & $S$ epidermidis & Wound \\
\hline & 2 & $S$ epidermidis & Blood culture \\
\hline & 3 & $S$ epidermidis & $\begin{array}{l}\text { Blood culture \& electrode tip } \\
\text { (septicaemia) }\end{array}$ \\
\hline & 4 & $S$ aureus & Pus in pocket \\
\hline & 5 & Sxylous & Blood culture \\
\hline & 6 & Enterobacter & Pus in pocket \\
\hline & 7 & Proteus mirabilis & Wound \\
\hline & & Acinetobacter & Wound \\
\hline & 8 & Aerococcus viridans & Blood culture \\
\hline & & Micrococcus spp & Valve \\
\hline & & Str viridans & $\begin{array}{l}\text { Tip of temporary pacing lead } \\
\text { (mitral valve endocarditis) }\end{array}$ \\
\hline \multirow[t]{2}{*}{ Infection and erosion } & 9 & $S$ aureus & Wound \\
\hline & 10 & Clostridium fallox & Wound \\
\hline Infection and fibrosis & 11 & $S$ epidermidis & Pocket \\
\hline \multirow[t]{9}{*}{ Erosion and infection } & 12 & Ps aeruginosa & Wound and electrode tip \\
\hline & 13 & Enterobacter agglomerus & Electrode tip \\
\hline & & Klebsiella & Wound \\
\hline & 14 & $S$ epidermidis & Electrode tip \\
\hline & 15 & $S$ epidermidis & Electrode tip \\
\hline & 16 & $S$ epidermidis & Blood culture \\
\hline & 17 & $S$ epidermidis & Pus from eroded lead \\
\hline & 18 & $S$ epidermidis & Electrode tip \\
\hline & & E coli & Wound \\
\hline \multirow[t]{2}{*}{ Wound never healed } & 19 & $S$ aureus & Wound \\
\hline & & Enterococci & Wound \\
\hline
\end{tabular}

All the positive microbiological findings among the patients in the study are shown. The complication in the left column was that recorded by the patients' physicians. procedure. Patients with temporary pacing electrodes were given a single dose of intravenous vancomycin one hour before the procedure. Skin preparation was with chlorhexidine gluconate and standard implantation procedures were used throughout.

OPERATORS

There were 32 operators during the period of this study (table 1). We classified experienced operators as suggested by Parsonnet et al. ${ }^{6}$ Fourteen were being trained (100 implants), seven were experienced operators $(>100$ implants), six were experienced operators but performed fewer than 12 procedures annually, and five were inexperienced and performed fewer than 12 procedures.

\section{STATISTICS}

Complication rates are expressed as the number of complications per 100 patients implanted, and 95\% confidence intervals for rates are given. Comparisons of rates are by Pearson $\chi^{2}$ statistic for contingency tables. Comparisons between rates for first implant and EUR have been adjusted for the proportion of single and dual chamber pacemakers in each group using the Mantel-Haenszel test. Probability (p) values less than 0.05 were considered significant.

\section{Results}

In total there were 50 patients who suffered complications, median age 72 years (range 20 to 97 years), $13(26 \%)$ female and $37(74 \%)$ male, giving a complication rate of $1.9 \%(95 \%$ confidence interval $1.36 \%$ to $2.44 \%$ ) occurring at a median of 11 months (range 51 days to 10 years) after unit implantation or replacement (table 2).

\section{COMPLICATIONS}

Infection

A clinical distinction was drawn between primary infection, with or without erosion (cases 1 to 11 , table 3 ), and erosion with superadded infection (cases 12 to 18 , table 3) as discussed below. The definition of infection in this context has been described previously. ${ }^{7}$ Of the 18 cases of infection, temporary transvenous pacing wires were present in only four patients at the time of pacemaker implantation. Positive microbiological results were found in $11(65 \%)$ and the organisms isolated are listed (cases 1 to 11 , table 3 ). The case of infection and fibrosis (case 11, table 3) was a soft tissue infection overlying the electrode near the clavicle, which was associated with a marked fibrous reaction.

\section{Erosion}

Among the 20 cases of erosion, 10 occurred after first implantation of a pacing system, giving a rate of $0.4 \%(0.14 \%$ to $0.66 \%)$, while 10 occurred following EUR, giving a rate of $4.5 \%$ $(1.86 \%$ to $7.14 \%)$. Thus erosions were significantly more common after EUR $(p<0.001)$. In 10 cases of mechanical erosion there was 
Table 4 Comparison of operator experience and complication rates

\begin{tabular}{llc}
\hline & $\begin{array}{l}\text { Complications/ } \\
\text { number of } \\
\text { patients }\end{array}$ & $\begin{array}{l}\text { Rates per patient } \\
\text { (95\% CI) }\end{array}$ \\
\hline $\begin{array}{l}\text { Experienced } \\
\text { Trainee }\end{array}$ & $8 / 871$ & $0.9 \%(0.3$ to 1.5$)$ \\
$\begin{array}{l}\text { Experienced } \\
\text { infrequent }\end{array}$ & $26 / 1422$ & $1.8 \%(1.1$ to 2.5$)$ \\
$\begin{array}{l}\text { Inexperienced } \\
{ }^{\mathrm{p}}<0.001 .\end{array}$ & $5 / 259$ & $1.9 \%(0.2$ to 3.6$)$ \\
& $7 / 37$ & $18.9 \%(6.0 \text { to } 31.8)^{\star}$ \\
\hline
\end{tabular}

clinical evidence of secondary infection. At least one organism was isolated in each of seven of these cases (cases 12 to 18 , table 3 ).

Electrode displacement

Three cases occurred, one in a dual chamber system after seven months and two in single chamber systems after two and 45 months.

\section{Electrode malfunction}

One patient with a single chamber unit had an insulation break affecting the lead at the header block after 10 years. Another patient had a malfunctioning lead after 16 months with a normally functioning generator, but no evidence of insulation break or lead fracture was discovered. No faulty connections occurred.

\section{Other complications}

Three patients developed heart failure and were upgraded to dual chamber systems. The pacemaker wound failed to heal in two cases (but presented after six weeks and are therefore included in this study of late complications) and one of these developed infection (case 19, table 3 ). One young patient with an active lifestyle requested repositioning of a superficially placed generator and another patient's generator was repositioned because of local pain.
Deaths

Two patients died in the late period after pacemaker implantation during the period covered by this study from a complication related to the pacemaker (late mortality $0.08 \%$ ). In both the implantation of a pacemaker was felt to be a significant factor. Both were complex cases.

One was a frail 88 year old man who underwent repositioning of a displaced lead from a VVI pacemaker eight weeks after implantation. Three weeks later he was readmitted with an infected system which was removed. He then developed klebsiella pneumonia and died in hospital from this complication.

The other death occurred in a 41 year old man with an atrial cardiomyopathy and atrioventricular block. Eight months after his fourth pacemaker procedure, infection developed and the entire system was removed under cardiopulmonary bypass. After surgery he suffered a hypovolaemic arrest and died despite urgent reoperation. At necropsy a sinus from the fibrous sheath which had enclosed the ventricular pacing lead was found to communicate with the aorta. It was surmised that when the lead was removed this created a channel from the aorta to the right atrium which led to catastrophic bleeding from the right atrial suture line which ruptured under systemic pressure. The pacing lead used in this case was not one subject to complications or product recalls previously.

PREDISPOSING FACTORS

Of the 50 complications, 46 were distributed among 32 operators at different stages of training and four could not be attributed to a specific individual (table 1). A median of one complication occurred among the operators (range 0 to 5). Only eight operators who performed implantations during the study period had no late complications at all.

Table 5 Previous or subsequent complications among patients with late complications

\begin{tabular}{|c|c|c|c|c|c|}
\hline Age & Sex & $\begin{array}{l}\text { Dual or } \\
\text { single } \\
\text { chamber }\end{array}$ & Early complications & $\begin{array}{l}\text { Complication in this } \\
\text { study }\end{array}$ & Subsequent complications \\
\hline 76 & M & $\mathrm{S}$ & Wound never healed & Same & None \\
\hline 68 & $M$ & S & Wound never healed & Infection of wound & None \\
\hline 66 & $\mathrm{~F}$ & S & Sinus excised 1 month & Infection & None \\
\hline 86 & M & $S(E U R)$ & $\begin{array}{l}\text { Superficial at } 1 \\
\text { month-repositioned deeper }\end{array}$ & Erosion & None \\
\hline 67 & $\mathrm{~F}$ & S (EUR) & $\begin{array}{l}\text { Abscess/infection-new } \\
\text { epicardial system }\end{array}$ & Erosion & None \\
\hline 67 & M & $S$ & $\begin{array}{l}\text { Febrile post-implant-oral } \\
\text { antibiotics only }\end{array}$ & Infection & None \\
\hline 74 & M & $\mathrm{D}$ & $\begin{array}{l}\text { Haematoma evacuated at } 1 \\
\text { month }\end{array}$ & Erosion + infection & None \\
\hline 71 & M & $\mathrm{S}$ & Lead displaced & Erosion + infection & None \\
\hline 75 & $\mathrm{~F}$ & $S$ & $\begin{array}{l}\text { Lead displaced at } \\
\text { implant-repositioned }\end{array}$ & Displaced lead & $\begin{array}{l}\text { New lead displaced again-further new lead } \\
\text { (active fixation) }\end{array}$ \\
\hline 41 & M & S (EUR) & None & Infection & Death (see text) \\
\hline 73 & M & $\mathrm{S}$ & None & Infection & Eroded 3 years later \\
\hline 93 & $\mathrm{~F}$ & S (EUR) & None & Erosion & New system also eroded-replaced \\
\hline 88 & M & S & None & Displaced lead & Death (see text) \\
\hline 46 & M & $\mathrm{S}$ & None & Infection + fibrosis & New system infected—new epicardial system \\
\hline 77 & M & $\mathrm{D}$ & None & Displaced lead & $\begin{array}{l}\text { New atrial lead displaced-replacement eroded, } \\
\text { therefore given VVI unit }\end{array}$ \\
\hline 53 & M & S (EUR) & None & Erosion & Recurrent staphylococcal infections \\
\hline 24 & $\mathrm{~F}$ & $\mathrm{D}$ & None & Pain around unit & $\begin{array}{l}\text { Further pain - new abdominal system, } \\
\text { endocardial lead }\end{array}$ \\
\hline
\end{tabular}

Patients are listed who had either a previous early complication or who developed a later complication, or both. The early complications here were those present within six weeks of pacemaker implant or EUR. The patients with wounds which failed to heal technically count as early complications only. However, these individuals did not present until after the six week period, one with infection.

$\mathrm{D}$, dual chamber; EUR, elective unit replacement; F, female; $M$, male; S, single chamber (VVI/R). 
Comparing complication rates with levels of operator experience it is clear that inexperienced operators not undertaking formal training had the highest complication rates (table 4).

There was no statistical difference in complication rates comparing dual with single chamber systems: 41 among 2220 single chamber systems $(1.8 \%(1.22 \%$ to $2.38 \%))$, and nine among 401 dual chamber systems $(2.2 \%$ $(0.74 \%$ to $3.66 \%)), p=0.59$. In this study no AAI pacemakers were installed and the comparison therefore reflects dual chamber versus ventricular sensing/pacing.

Sixteen $(32 \%)$ of the late complications occurred in patients undergoing EUR, though EUR made up only $9 \%$ (245 of 2621 ) of the total procedures performed during the 11 year period studied (table 3 ). The rate of complications was significantly higher in patients having EURs compared with those receiving their first implant, at $6.5 \%(3.3 \%$ to $9.7 \%)$ v $1.4 \%(0.9 \%$ to $1.9 \%), \mathrm{p}<0.001$.

Data regarding the presence of a temporary pacing electrode present at the time of implantation are only available from September 1989, after which 2061 implants were performed. Of these, 642 had a temporary wire and two developed complications $(0.8 \% \quad(0.1 \%$ to $1.5 \%)$ ); the remaining 1419 did not have a temporary wire and 22 developed complications $(1.6 \%(0.9 \%$ to $2.2 \%))$. This difference was not statistically significant.

Early complications had occurred in nine patients, of whom one went on to have further complications, along with eight other individuals (table 5).

\section{Discussion}

Late complications requiring operative intervention following pacemaker implantation are remarkably rare. We have documented an overall complication rate of only $1.9 \%$ over 11 years, which compares favourably with the rate of early complications described by ourselves and others. Operator experience appears to influence the likelihood of late complications (as it does early complications). ${ }^{3}$ We are not aware of any other large series that has specifically addressed late complication rates, though reports of all the complications which occurred during long term follow up have been published. ${ }^{89}$

The most frequent problems in studies of early complications have been lead displacement, wound infection and erosion, and haematoma. ${ }^{124}$ The dominant complications in this study were erosion and infection.

The rate of erosion, the most common complication in our study, was only $0.8 \%$. This is much lower than the $3.7 \%$ reported by Griffith et al for all cases of erosion (early and late) over a 10 year period. ${ }^{10}$ The discrepancy may at least partly be explained by the fact that we restricted our analysis to late complications. There may also have been differences in implantation technique, degree of experience among the operators involved, and the patient population, and varying interpretation of the diagnostic overlap between infection and ero- sion. The incidence of erosion in our study and that of Griffith et al contrasts with the experience of Hill, who reported no cases of erosion at all among 589 patients over 14 years. ${ }^{8}$ More recently, the 1993 survey of pacing in the United States suggested that $1.7 \%$ of all replacement pulse generators took place owing to erosion or infection. ${ }^{11}$

Pacemaker erosion may be caused by mechanical factors, or may occur as a result of system infection with subsequent erosion. The clinical overlap between patients with mechanical erosion with superadded infection and those with primary infection and subsequent erosion is important because there is evidence that it is possible to reimplant pacemakers where erosion is not caused by infection. The most significant factor predicting success of reimplantation is the absence of bacterial growth. ${ }^{10}$

The high incidence of infection in our cases of erosion therefore indicates a need for careful clinical appraisal. Microbiological confirmation of the presence of infecting organisms was detected in 11 of 17 cases of primary infection $(65 \%)$ and in seven of 10 cases of mechanical erosion with infection $(70 \%)$. Organisms were also detected in one of the two cases where patients reported failure of the wound to heal. It therefore appears that cases of erosion with clinical evidence of infection are as likely to be associated with positive microbiological findings as are those where there is a clinical suspicion of infection alone.

The infection rate in our study $(0.55 \%$ after first implant) compares with $0.5 \%$ in Hill's study, ${ }^{8} 0.69 \%$ in a report by Matsuura et al, ${ }^{9}$ and $0.9 \%$ in Mueller's study. ${ }^{12}$ In a large study by Frame et al concerning 5089 patients over a 20 year period the infection rate was $1.06 \% .^{13}$ The infection rate in the current study is lower than in most of these other reports, probably because early cases were excluded. However, a significant role for infection was found in the majority of late complications, the most common organisms isolated being staphylococcal species. The total number of cases associated with clinical evidence of infection in this study was 27 (1.03\%: 17 cases of infection, two of infection leading to erosion, and eight of probable mechanical erosion and superadded infection), suggesting that this is by far the most important factor in late complications.

Intravenous antibiotic administration as prophylaxis against postprocedure infection has recently been shown to be effective in a large randomised controlled trial with follow up for a mean period of 19 months, though one previous large trial showed no benefit and another only a small reduction in infections. ${ }^{14-16}$ Therefore, though the role of prophylaxis in the prevention of infection in the longer term is not clear, we continue to use routine oral antibiotic prophylaxis, with intravenous antibiotics in cases with perceived additional risk (such as the presence of a temporary pacing wire or early reoperation for displaced leads).

The rate of complications following elective unit replacement was disappointingly high at $6.5 \%$. All of these occurred in patients with 
single chamber units, which may simply reflect the low rate of elective unit replacements for dual chamber systems performed during this study (10 in all). The majority of cases involved erosion, which appeared to be more common in these patients than in those undergoing first implantation. Closer attention to the surgical technique during elective unit replacement and to subsequent follow up of these patients might therefore be appropriate. Elective unit replacement may best be performed by experienced operators.

Though recent studies have shown complication rates were no higher for dual than for single chamber systems, ${ }^{412}$ our study suggests that late complications are more often observed with dual chamber systems, in keeping with a previous study. ${ }^{3}$ We speculate that subsequent studies may show that late complications are most commonly observed after elective unit replacement of dual chamber systems. The presence of a temporary wire did not have a significant impact on late complications, which contrasts with the deleterious effect observed with early complication rates. ${ }^{3}$ Operator experience does seem to be an important factor in both early and late complication rates. ${ }^{2}{ }^{10}$

Early complications, which did seem to influence subsequent events, occurred in nine patients (18\%) (table 5), though in two the early complication was the same as the late complication (wound never healed). One of these patients had further problems of a similar nature and eight other patients (nine in all, $18 \%$ ) developed later complications, having had no early problems. These data imply a need for careful follow up, since nearly one fifth of patients will go on to have further problems after a complication.

In conclusion, the rate of late complications after first implant of a permanent pacemaker system is $1.4 \%$, the majority of these relating to infection and erosion. Inexperienced operators have a higher complication rate than experienced operators or those undergoing training who go on to become experienced operators.
The rate of late complications following elective unit replacement is much higher and usually a result of erosion. Our results suggest that the total complication rate (early plus late) following permanent pacemaker implantation at a large regional cardiothoracic centre is around $3.5 \%$.

1 Hall JA, Grace AA, Newell SA, et al. Prospective evaluation of the early complications of 1136 single chamber permanent pacemaker implantations. Eur $\mathcal{F}$ Cardiac Pacing Electrophysiol 1993;3:14-20.

2 Mugica J, Ritter P, Lazarus B. Evolution of early complications after ventricular single chamber pacemaker implants in a specialised centre: 9483 patients over 24 years. Eur $\mathcal{f}$ Cardiac Pacing Electrophysiol 1993;3:21-6.

3 Chauhan A, Grace AA, Newell SA, et al. Early complications after dual chamber versus single chamber pacemaker implantation. Pacing Clin Electrophysiol 1994;17:2012-5.

4 Aggarwal RK, Connelly DT, Ray SG, et al. Early complications of permanent pacemaker implantation: no difference between dual and

5 Parsonnet V, Furman S, Smyth NPD. Implantable cardiac Parsonnet V, Furman S, Smyth NPD. Implantable cardiac
pacemakers: status report and resource guidelines, Interpacemakers: status report and resource guidelines, InterSociety Commission on Heation 1974;50:21-35.
Circulation

Circulation 1974;50:21-35.
6 Parsonnet V, Bernstein A, Lindsay B. Pacemaker implantation complication rates: an analysis of some contributing factors. F Am Coll Cardiol 1989;13:917-21.

7 Choo MH, Holmes DB, Gersh BJ, et al. Permanent pacemaker infections: characterization and management. Am f Cardiol 1981;48:559-64.

8 Hill PE. Complications of permanent transvenous cardiac pacing: a 14-year review of all transvenous pacemakers inserted at one community hospital. Pacing Clin Electrophysiol 1987;10:564-70.

9 Matsuura Y, Yamashina H, Higo M, et al. Analysis of complications of permanent transvenous implantable cardiac pacemakers related to operative and postoperative management in 717 consecutive cases. Hiroshima f Med Sci 1990; 39:131-7.

10 Griffith MJ, Mounsey JP, Bexton RS, et al. Mechanical, but not infective, pacemaker erosion may be successfully managed by re-implantation of pacemakers. Br Heart $\mathcal{f}$ 1994;71:202-5

11 Bernstein AD, Parsonnet V. Survey of cardiac pacing and defibrillation in the United States in 1993. Am f Cardiol 1996;78:187-96.

12 Mueller X, Sadeghi H, Kappenberger L. Complications after single versus dual chamber pacemaker implantation. Pacing Clin Electrophysiol 1990;13:711-14.

13 Frame R, Brodman RF, Furman SF, et al. Surgical removal of infected transvenous pacemaker leads. Pacing Clin Electrophysiol 1993;16:2343-8.

14 Mounsey JP, Griffith MJ, Tynan M, et al. Antibiotic prophylaxis in permanent pacemaker implantation: a prospective laxis in permanent pacemaker implantation:

15 Ramsdale DR, Charles RG, Rowlands DB, et al. Antibiotic prophylaxis for pacemaker implantation: a prospective ranprophylaxis for pacemaker implantation: a prospective
domised trial. Pacing Clin Electrophysiol 1984;7:844-9.

16 Muers MF, Arnold AG, Sleight P. Prophylactic antibiotics for cardiac pacemaker implantation: a prospective trial. $\mathrm{Br}$ Heart f 1981;46:539-44. 